\title{
The Design of Smart sphygmomanometer based on Android Mobile Device
}

\author{
Gaoxu Deng \\ Shanghai University of \\ Engineering Science \\ Songjiang Shanghai \\ 201620, China
}

\author{
Chen Deng \\ Shanghai University of \\ Engineering Science \\ Songjiang Shanghai \\ 201620, China
}

\author{
Yiming Wang \\ Shanghai University of \\ Engineering Science \\ Songjiang Shanghai \\ 201620, China
}

\begin{abstract}
Faced with a growing number of hypertensive patients blood pressure measurement needs, this paper designed the intelligent wearable device based on Android phones. Mainly introduces the design and implementation of hardware and software of the Android mobile phones and STM32 as the core, individual blood pressure and heart rate control and measurement. Implements of the Android WiFi control of blood pressure, blood pressure measurement and abnormal warning $\mathrm{s}$ a good way to meet the demand for the hypertension patients on blood pressure measuremen, has the characteristics of high performance, low cost and low power consumption.
\end{abstract}

\section{General Terms}

Android APP Development

\section{Keywords}

Android APP Development,Wearable Device,WiFi Wireless Communications,Data Collection.

\section{INTRODUCTION}

With the continuous expansion of hypertensive patients in our country, the people increased the importance of high blood pressure . In the age of the Internet of things, Mobile phone has more and more applied on the Internet of things as a basic platform. The Internet of things based on smart phone has been used in the field of environmental monitoring, intelligent security monitoring, intelligent transportation and telemedicine, mobile payment, and other fields. The Android mobile operating system is based on the Linux kernel, open source code. .The development of the APP is mainly aimed at the application layer design, using the Java language in Android development platform for development, and uses the Android mobile phone in the programming interface for communication with the device.

Based on the Android smartphone,this paper combined with embedded system technology, sensor technology, supplemented by WiFi access technology design of a wearable device, through the development of smart phone APP to realize the wireless control of blood pressure, and achieves real-time to determine hypertension disease and alarm.

\section{THE INTRODUCTION OF ANDROID DEVELOPMENT TECHNOLOGY}

\subsection{The Android system framework}

Android USES the layered architecture, from top to bottom, respectively the Application layer (Application), the Application Framework layer (Application Framework), the system is running (including Libraries, Android Runtime), the Kernel (Linux Kernel).Android's four components are respectively the activity, service, control the provider, broadcastreceiver. And the content view is the view hierarchy of the root node view.

1 , activity

Each activity is inherited from the parent class of Android. The activity window display view can call activity. setContentView () method to set.

The Activity's booting process as shown in Fig 1:

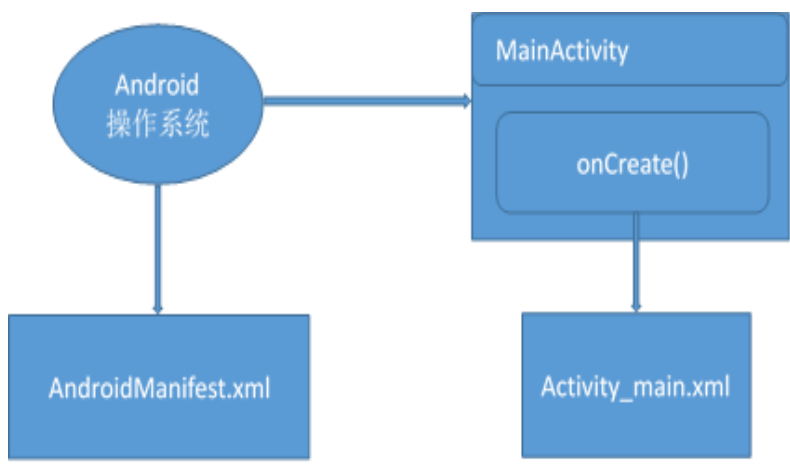

Fig 1: The Activity's booting process

2, service

Each service is inherited from the parent class, service.The service start after running in the background, no user interface. Service, for instance, may be network data to be loaded in dealing with other things, or playing MP3, or performing some time consuming operations, and going back the computing results to the activity display in the user interface.

The Applications can bind to connect a running service, after the connection is successful, and can offer interface and communication through this service. Similar to the activity and other components, the service runs in the main thread of the application process, to ensure that no other components,

the user interface block,performing time-consuming tasks need to derive a worker thread.

\section{3, broadcastreceiver}

All of the broadcast receiver inherited from the parent class, broadcastReceiver. Most of the broadcast notification is produced by the former. When the battery is too low, for example, users receive text messages to remind, or user to change the default Settings, and so on. Developers can also push broadcast notice in the application, such as to inform other applications network data been loaded. Developers can also push broadcast notice in the application, such as to inform other applications network data has been loaded. 
4, content provider

Content provider, inherited from the parent class, ContentProvider.It provides a standard interface for other application calls and data access control. These Shared data can be stored file system or SQLite database.

\subsection{Android network programming}

Based on the Android network programming,in general,using the socket network data communication between devices, common Socket network programming including TCP and UDP protocol.

Socket is used to describe a device on the Internet in a process, or description of an application, the socket consists of two parts, the IP address and port number, IP address format for: 192.168.0.0.3, port number for four digits, such as 8888 .

In general, the socket communication includes both server and client The Server is responsible for monitoring and request response to a request for develop port, the client to initiate connection requests to the Server port.. When the client and the server request response success, can generate a socket object after connection is established, and the data to communicate via the socket.

The basic process of using socket for data exchange in Androi $\mathrm{d}$ is as follows:

\section{ServerSocket}

When building the server,you need using a ServerSocket object, bounding to the listener port number, and starting listening port, after received a connection request from the client, then using the method of serversocket.accept ()to returns a connected socket object.

\section{Socket}

Establishing a client required using socket object,socket in the initialization need to specify the IP address and port number of the server firstly, connected to be successful that can be returned to the side of a socket object of the server, the end of server end and client can be connected and exchanged data; if they failed, an exception will be thrown.

\section{IO data stream interaction}

After establishing the socket,you can use OutputStream classes and InputStream classes in the IO stream to exchange data .

When we need to operate the data, you can use the method of get OutputStream () to obtain the data stream ,and operation; when you need to fetch data from the stream, you can use the

method of Socket.get InputStream () to obtain the data flow and then make related operation.

\subsection{The embedded system technology}

Embedded hardware system consists of embedded processor and related support hardware and peripheral interface circuit etc.

This design's STM32 processor has the following features:

(1) The 32-bit Cortex-M3 CPU, the highest working frequency $72 \mathrm{MHZ}$.

(2) The Flash capacity is 256 k, 64 k SRAM capacity.

(3) Single cycle hardware multiplication and division.

(4) Dormancy, stop, three kinds of low power consumption standby mode.

(5) 12 channel DMA controller, support the ADC and DAC, SPI, I2C, UART peripherals.

(6) Two independent grade 12 us analog-to-digital conversion module (16 channels), measurement range of $0 \sim 3.3 \mathrm{~V}$

(7) Equipped with nested interrupt vector controller, convenient application code transplantation on CortexM3 platforms.

(8) 112 general I/O port (8), each group of ports can be mapped to 16 external interrupt vector.

(9) 16 bit general timer, two senior plus Systick timer, RTC.

(10) STM32 core through special instruction bus CortexM3,connect with Flash memory, the system bus and data bus and AHB (advanced high-speed bus), external devices using two APB (bus) advanced equipment is linked, every piece of APB bus is connected to the AHB bus matrix.

(11) STM32 processor internal structure as shown in Fig 2:

\section{Description of system overall design}

This system is based on the smart Android mobile blood pressure monitoring system, This paper includes the following two parts:

1. The measurement system.

2. The remote control.

The application layer of this system through the WiFi control of blood pressure heart rate will be shown on the measurement data to return to the interface, and compares with the international standard health parameters and voice warning.

The design of the system structure as shown in Fig $\mathbf{3}$ : 


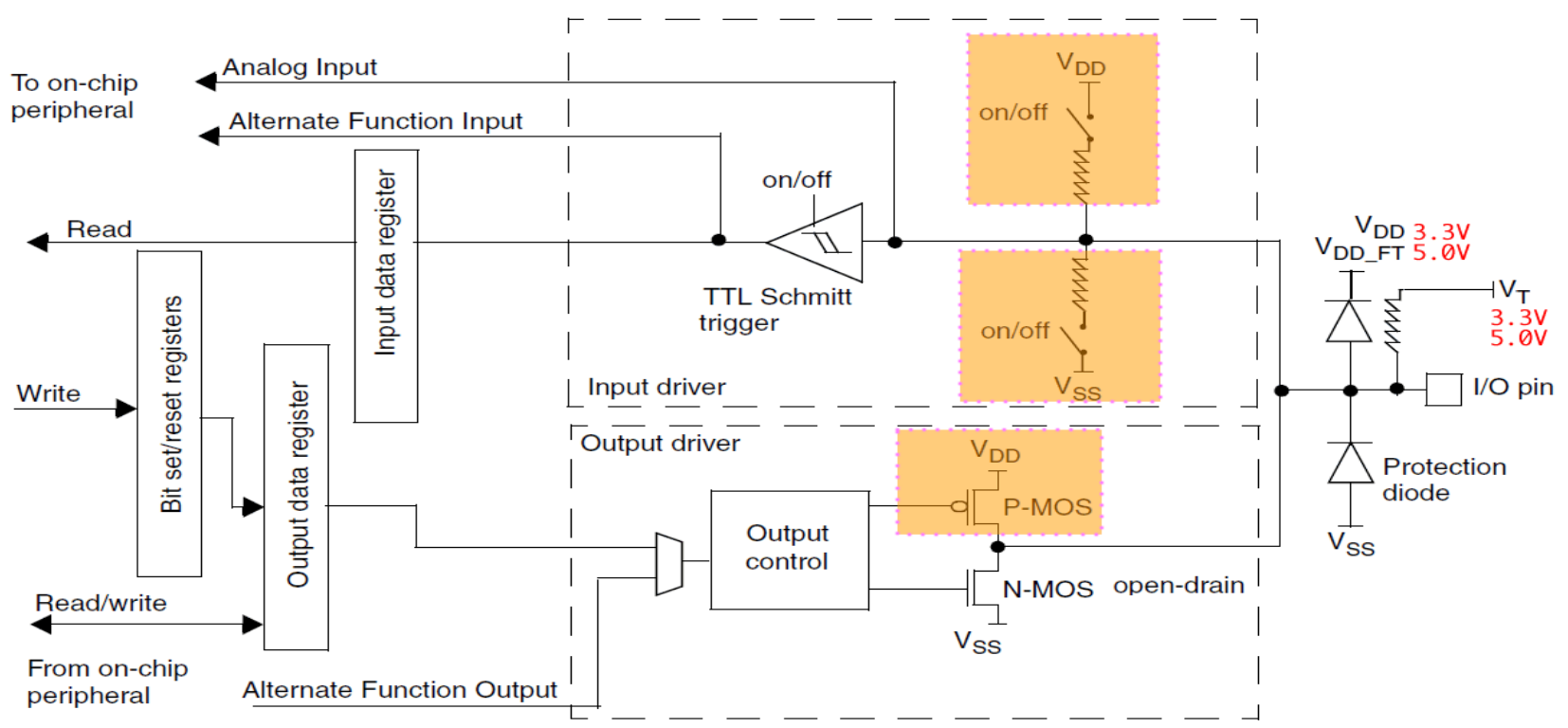

Fig 2: STM32 processor internal structure

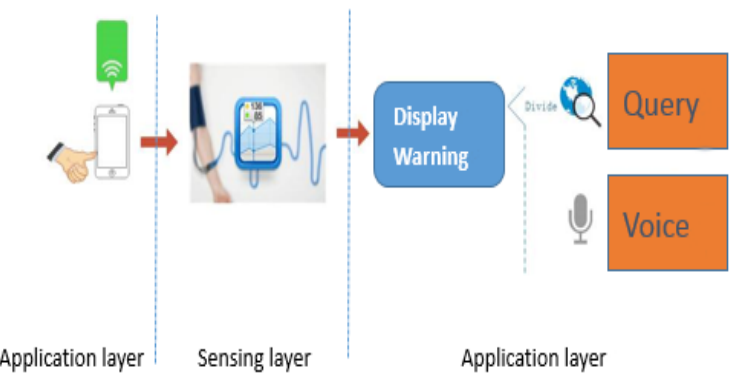

Fig 3: The design of the system structure

The application layer - mobile client:

The application layer of this system mainly based on Android smartphone development, mobile phone, as the user interaction with the system interface, plays an important role. Project 's most important task is based on the Android mobile phone to develop a friendly interactive interface, can also provide users with real-time control wearable device for blood pressure measurement and display.

The application level of the specific features include three aspects:

1. Open WiFi connection through mobile phone interface button control, with the communication of the microprocessor.

2. By starting measuring data acquisition cuff button control device, waiting for the end of the measurement data sent via wi-fi network in the form of data flow to mobile phones, handling by the program will display the data on the mobile phone interface.

3. The measurement data are implemented by software with the international standard blood pressure data than to prompt the current blood pressure and heart rate is normal voice health tips.

The APP human-computer interaction interface as shown in Fig 4:

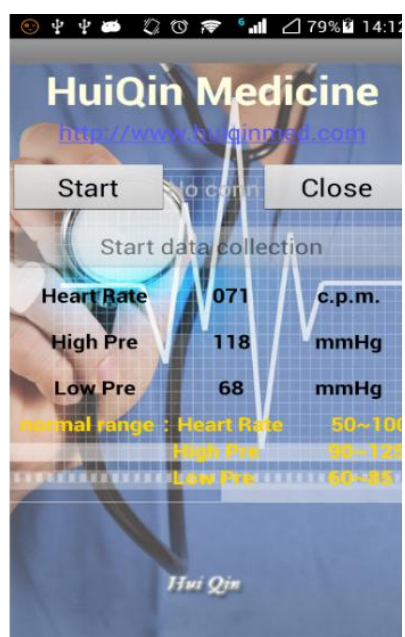

Fig 4: The APP human-computer interaction interface

The design of the APP in eclipse is commonly used Android and Java development platform, the platform has the advantage of simulating the Android emulator and connecting directly to the corresponding real-time debugging system version of the Android mobile phone.

Mainly for the res resource file and the design of SRC the source program files.

(1) The Android 4.4

Project selection is the Android 4.4 API, the package deposit is related to the project under the bottom of the bag.

(2) The Android Pivate Lbraries

Android Private Libraries reference is a third-party jar package, for Private reference, add jars to the libs, the system automatically put the jar is loaded into the Android Private Libraries.

(3) The src

Deposit in the SRC folder is "business logic code", it is. "Java" suffix at the end of the file. 
(4) The Gen

Gen folder to store the index file is automatically generated by the project, such as the res $\backslash$ layout $\backslash$ the main XML defined in a text boxes (Textview) with id "@ + id/TextView1" in R.j ava to generate the corresponding code: "public static int TextView1 $=0$ x7f050000;"

(5) Assets

Assets folder store the native file not compiled processing, indicates that the file in folder not like the XML file is compiled, the main store is some images, HTML, js, CSS files, etc.

(6) Bin

Bin folder store the binary executable file.

(7) Libs

Libs folder support installation files are kept in the file.

(8) Res

Res folder store a resource file, the file with the assets folder to store resources file differs: the Res store the native files need to compile processing, file by default have drawable hdpi, drawable mdpi, drawable - ldpi, layout and values folders.

Among them, the start icon is simple,be handled by Photoshop editing the pixel size of different PNG format images added to the res drawable resource file in the AndroidManifest bin file. The XML for the android: icon, so as to realize the change of startup screen.

APP interface of human-computer interaction interface write mostly in res file layout in the implementation of the resources, mainly through the use of eclipse controls to implement, and then modify the layout folder activity_main. The relevant application of XML, mainly include the button button controls, edit edit control, textview text display control and control the layout of the control.

PC man-machine interactive interface design is Android installation package apk Scrollview, the overall layout of Android first class layout_width and layout_height set to fill_parent, scrollbars set to vertical, linerlayout is to control layout, main setting properties for fill_parent layout_width and layout_height is match_parent, orientation is vertical.

The rest of the controls such as textview and a button control is directly put into the linerlayout, each control has its own unique id, other properties to the corresponding design meet the need of design.

The phone APP and STM32 use local WiFi to communicate, mainly in the SRC folder in the MainActivity. In Java implementation, the main socket wireless Internet connection in a new thread, use the try - catch catch exceptions unknown abnormal UnknownHostException and IO stream IOException.

If caught, then send the information to close the application, handle to set the format for sendEmptyMessage (0), the socket of the IP address and port number in the InetSocketAddress Settings, and instantiated as objects, the key lies in the input and output of data flow, set up as follows:

isa $=$ new InetSocketAddress("192.168.10.3", 5050); C_socket.connect(isa, 1000);
Data_out $=$ new PrintStream $\left(C \_s o c k e t . g e t O u t p u t S t r e a m()\right)$;

Client_s = C_socket.getInputStream();

Data_in = new DataInputStream(Client_s);

Perception layer-data acquisition terminal

The perception layer of this system mainly uses the wearable sleeve type pressure sensor, microprocessor by parsing a mobile phone to send commands to control blood pressure cuff on the startup and shutdown.

Application layer and layer perception of WiFi interactive data processing is mainly transmitted in the form of input and output data flow, perception layer when sending data to deal with the data format, respectively, in the format of the AT + to send, in the MainActivity. Java will input data flow interception of useful data into the form of a string, and through the switch case select statement parsing choose different kinds of data and command.

Android mobile terminal data parsing and processing is done in a separate thread, inherit the parent thread, in function mainly using input and output data stream Data_in, Data_out read method in the form of 16 bytes of data read, into data information and send the key operation is the following code.

Data_MSG = new String(read_buff, 0, length, "gb2312");

handler.sendEmptyMessage(2);

Data acquisition running instance:

1. Blood pressure control implement is the humancomputer interaction interface as shown in Fig $\mathbf{5}$ on the control of blood pressure.

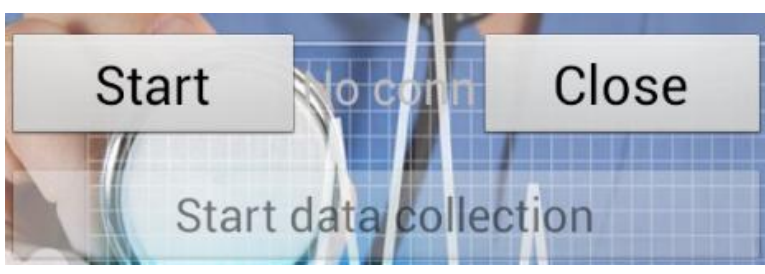

Fig 5: Blood pressure control interface

1. After measuring the interface of the blood pressure and heart rate data display as shown in Fig 6 on the mobile phone interface, finished on the wisdom of the wearable device in a wireless control and measurement:

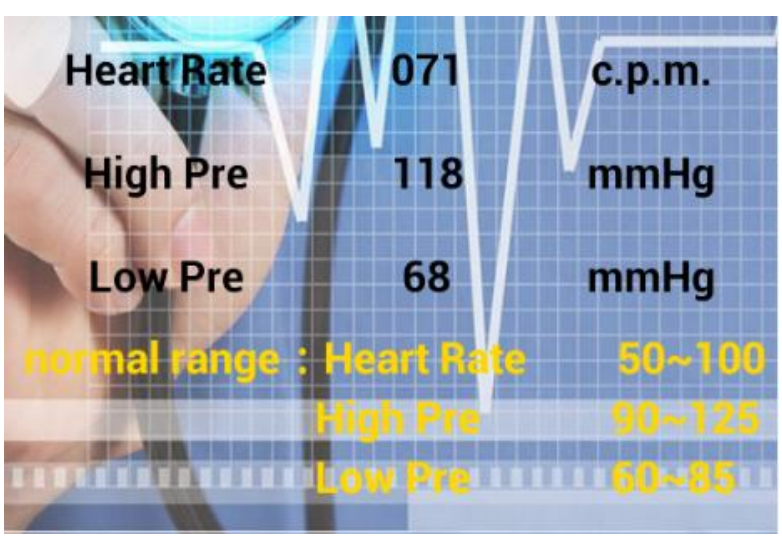

Fig 6: The result of the experiment test 


\section{CONCLUSION}

In this paper, a design and implementation based on the the Android mobile device. It can pass the WiFi real-time control of wearable device for the user's blood pressure and heart rate collection, especially for the elderly, such as high blood pressure patients,it can accurately grasp their own blood pressure condition, wearable cuff blood pressure can be directly and wear in the user's arm, the control of mobile phone APP can reach anywhere at any time of the measurement of blood pressure and heart rate. Data collection using the embedded system more popular STM32 core board, has a strong ability to control and process, and can be very fast to control sensors, ensure the accuracy of the data acquisition, it has the characteristics of high performance, low cost, low power consumption, great market value.

\section{ACKNOWLEDGMENTS}

I would like to show my deepest gratitude to my supervisor, Dr.Deng chen, a respectable, responsible and resourceful scholar, who has provided me with valuable guidance in every stage of the writing of this thesis. Without her enlightening instruction, impressive kindness and patience, I could not have completed my thesis. Her keen and vigorous academic observation enlightens me not only in this thesis but also in my future study.

\section{REFERENCES}

[1] Chaoxin Lin. Internet monitoring system based on Android mobile phone research[D]. Xiamen University.2014.

[2] Dakun Zhai,Chuncha Chen. The Android project development steps [M]. Mechanical industry press.2010.
[3] Tingting Wang. Based on STM32W intelligent environment monitoring system[D]. Jiangsu university of science and technology.2013.

[4] Xiaoxiao Liang,Huibing Qin. Based on the realization of the STM32 WiFi local area control module [J]. Science and technology information. 2015.

[5] Kunjia Zhou,Chen Bo,Li Chen. Based on the STM32 data collector design and implementation of personal health and environment [J]. Computer knowledge and technology.2015.

[6] Wang Chao. Based on the STM32 home monitoring system design and implementation [D]. Wuhan university of technology. 2014.

[7] Jingming Xie. Android mobile development tutorial [M]. People's posts and telecommunications publishing house. 2013.

[8] McCallum, G.: EGADSS: A Clinical Decision Support System for use in a Service-oriented Architecture. MSc. Thesis, Computer Science, University of Victoria (2006).

[9] C. Donn, S. Schwab, "Using the Vehicle-in-the-Loop (VIL) Approach for Model Based Development of Advanced Driver Assistance Systems," presented at the JSAE Annual Congress (Spring), Pacifico Yokohama, 2014.

[10] M. R. Zofka, R. Kohlhaas, T. Schamm, J. M. Zöllner: Semivirtual Simulations for the Evaluation of Visionbased ADAS, Proceedings of IEEE Intelligent Vehicles Symposium,2014. 